\title{
Multiple Sensor Fault Detection in Heat Exchanger Systems
}

\author{
Abdelwahab Aïtouche*,Didier Maquin"*, Frédéric Busson” \\ * Laboratoire d'Automatique et d'Informatique Industrielle de Lille \\ Hautes Etudes Industrielles \\ 13, rue de Toul, 59046 LILLE (France)
}

Tel: +33328384858 - Fax: +33 32028384804 - Email: aitouche@hei.fr

${ }^{* *}$ Centre de Recherche en Automatique de Nancy

2, Avenue de la Forêt de Haye

54516 VANDOEUVRE-LES-NANCY (France)

Tel: +33 383595683 - Fax: +33383595644 - Email: dmaquin@ensem.u-nancy.fr

\begin{abstract}
A two stage strategy for fault detection, isolation and estimation of multiple gross measurement errors (faults) in a class of systems described simultaneously by linear and bilinear equations is presented. The first stage consists in the detection and isolation of gross measurement errors intervening only in the linear equations. The second stage deals with the detection and isolation of gross measurement errors intervening on the bilinear equations by using corrected measurements of the preceding stage. A GLR method is employed to detect and to isolate the faults. An application of the proposed strategy is presented for a heat exchanger system for which the process model consists of a set of linear and bilinear equations. Simulations were performed to test the efficiency of the proposed method.
\end{abstract}

\section{Introduction}

Systems described simultaneously by linear and bilinear equations constitute an important potential area for data reconciliation techniques. This type of model structure is often used when describing a flow process (mineral or chemical industry, oil refinery) by material and/or energy balance equations, and can be found in heat exchangers.

The increasing importance of heat exchangers in the industry is not to be demonstrated. A set of interconnected heat exchangers may be described by a static linear-bilinear model obtained by the writing of mass and thermal balances of these exchangers.

We were interested in using fault detection and isolation techniques to validate the data provided by sensors before using them for controlling the process.

Classically, this data validation or data reconciliation is divided in the following steps : preliminary system decomposition according to the observability, detection, isolation, estimation of faults and data reconciliation.

In the last two decades, various methods for detection and isolation of gross errors in process data have been proposed including generalized likelihood ratio method (GLR) [1], measurement test method [2], parity space method [3] and pseudo nodes method [4].

The aim of this paper is to extend the utilization of the GLR test to detect and isolate sensor faults for a class of systems described simultaneously by linear (mass balance) and bilinear equations (thermal balance). The chosen application is a heat exchanger system.

The proposed method comprises two stages. The first stage is the generation of linear residuals using a classical method like these cited previously. Afterwards, the detection, isolation, identification and correction of the measurements intervening only in linear equations are done.

The second stage involves the generation of bilinear residuals. The GLR test is applied to generate bilinear residuals by using validated measurements of the preceding stage. This test allows the detection of measurement faults that intervene only in bilinear equations. The phases of isolation, identification and correction can then be driven in analogous manner to the preceding stage.

This paper is organized as follows. In the second section, the hypotheses which are necessary for developing the proposed method are described. The third section is dedicated to the description of the used models. Afterwards, the residuals generation is described in the following section. The fifth section remembers fault detection and isolation based on the use of GLR test and 
describes the proposed strategy. Then, a simple simulated example illustrates the method.

\section{Hypotheses}

The following hypotheses are assumed to be verified:

- the models (linear and bilinear) are exact,

- the measurement errors are not correlated and are normally distributed with zero mean,

- the variances of these errors are known or estimated.

In the following, we will denote by $Q$ the vector of flow measurements and by $T$ the vector of temperature measurement. The vector $Q$, the vector of measurement errors $\varepsilon_{Q}$ and the vector of flow sensor faults $d_{Q}$ are connected with the vector of true values $Q^{*}$ by the model:

$$
Q=Q^{*}+\varepsilon_{Q}+d_{Q}
$$

The vector $T$, the vector of measurement errors $\varepsilon_{T}$ and the vector of temperature sensor faults $d_{T}$ are also connected with the vector of true values $T^{*}$ by a similar model:

$$
T=T^{*}+\varepsilon_{T}+d_{T}
$$

All these vectors are of dimension $v \times 1$. Moreover, let us denote $V_{Q}$ (respectively $V_{T}$ ) the variance-covariance matrix of $\varepsilon_{Q}$ (respectively $\varepsilon_{T}$ ).

\section{Representation of heat exchanger system}

A heat exchanger unit can be modeled by a network represented in figure 1; four streams are considered with measurements of flow and temperature.

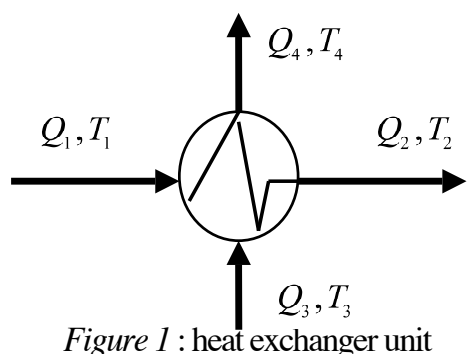

The incidence matrix $M_{Q}$ of dimension $m \times v$ relative to linear equations can be written as:

$$
M_{Q}=\left(\begin{array}{cccc}
1 & -1 & 0 & 0 \\
0 & 0 & 1 & -1
\end{array}\right)
$$

In this matrix, a "1" indicates an input variable and "-1" indicates an output variable. So the linear part of the mathematical model is written under the exact structural from:

$$
M_{Q} Q^{*}=0
$$

The true values of flows and temperatures are linked by the following bilinear constraints:

$$
M_{T} D_{Q^{*}} T^{*}=0
$$

where $M_{T}$ is an incidence matrix of dimension $n \times v$ relative to bilinear equations and $D_{Q^{*}}$ is a diagonal matrix whose diagonal is formed of the components of the vector of the true values of flows. This type of notation will be used throughout this paper.

In the case of a simple heat exchanger unit, $M_{T}$ is given by:

$$
M_{T}=\left(\begin{array}{llll}
1 & -1 & 1 & -1
\end{array}\right)
$$

\section{Residual generation}

The vector of flow measurements $Q$ does not verify the constraint equation (3). Let us define the vector of linear residuals $R$ of dimension $m$ :

$$
R=M_{Q} Q
$$

In the absence of flow faults and under the previous hypothesis relative to the measurement errors, the vector $R$ is normally distributed with zero mean and a variance matrix:

$$
H_{R}=M_{Q} V_{Q} M_{Q}^{T}
$$

In the presence of flow faults, the vector $R$ is normally distributed with a mean equal to $M_{Q} d_{Q}$ and a variance matrix $H_{R}$ which is identical to the previous case.

The vector of flow measurements and the vector of temperature measurements does not verify the bilinear equation (4). In the same manner that in the linear case, a vector of bilinear residuals may be defined:

$$
W=M_{T} D_{Q} T
$$

where $D_{Q}$ is a diagonal matrix whose diagonal is formed of components of vector $Q$.

In the absence of faults, the vector $W$ is normally distributed with zero mean and a variance matrix:

$$
H_{W}=M_{T} D_{Q^{*}} V_{T} D_{Q^{*}} M_{T}^{T}
$$


while in the presence of faults, its mean and variance matrices become:

$$
\begin{aligned}
& \operatorname{Esp}(W)=M_{T}\left(D_{T^{*}} d_{Q}+D_{\left(Q^{*}+d_{Q}\right)} d_{T}\right) \\
& \operatorname{Var}(W)=M_{T} A M_{T}^{T}
\end{aligned}
$$

with $A=D_{Q^{*}} V_{T} D_{Q^{*}}+D_{T^{*}} V_{Q} D_{T^{*}}+V_{Q} V_{T}$

Any diagonal matrix $D$. is constituted by the entries of the vector • .

As we have observed, the expected value of the vector $W$ depends simultaneously on the magnitude of flow and temperature measurement faults and true magnitudes of these two variables. In the presence of flow and temperature measurement faults, it will be practically impossible to isolate the flow or the temperature that is faulty directly. In addition the matrix of variance-covariance of residuals depends on unknown true magnitudes. To detect flow and temperature measurement faults, it is therefore necessary to estimate this matrix of variancecovariance.

The aim of the following is to generate a new residual of bilinear constraint which is insensitive to flow measurement faults. The flow measurements are then replaced by their estimation and a new residual vector $\hat{W}$ may be written as:

$$
\hat{W}=M_{T} D_{\hat{Q}}^{T}
$$

With this transformation, the detection-isolation of temperature measurement faults may be done as for linear systems as the matrices $M_{T}$ and $D_{\hat{Q}}$ are perfectly known.

\section{Detection by GLR method}

Thereafter, we consider first the case where only a single temperature sensor is faulty and next, we will extend the method to the case of multiple faults.

In the case of a single temperature fault, equation (2) becomes:

$$
T=T^{*}+\varepsilon_{T}+b_{T} e_{j}
$$

where $e_{j}$ is a vector of dimension $v$ containing 1 in position $j$ and zero elsewhere and $b_{T}$ is the unknown magnitude of temperature fault.

The problem of detection, isolation and correction of this fault consists in determining the faulty measurement $j$ and the magnitude of this fault.

The mathematical expectation of $\hat{W}$ is:

$$
\operatorname{Esp}(\hat{W})=b_{T} f_{j}
$$

with

$$
f_{j}=M_{T} D_{\hat{Q}} e_{j}
$$

Its variance matrix is:

$$
\hat{H}_{W}=M_{T} D_{\hat{Q}} V_{T} D_{\hat{Q}} M_{T}^{T}
$$

If we consider that flow estimations are closed to their true values, the matrix of variance-covariance (13) may be considered as a good estimation of (8).

So as to detect sensor faults, a test of hypotheses may be implemented. The two following hypotheses focus on the value of the expected value of bilinear constraint residuals.

$$
\begin{array}{ll}
H_{0}: \operatorname{Esp}(\hat{W})=0 & \text { no temperature fault is present. } \\
H_{1}: \operatorname{Esp}(\hat{W})=b_{T} f_{j} & \text { a temperature fault is present. }
\end{array}
$$

In order to test these hypotheses, using the normal probability density of $\hat{W}$, the likelihood ratio of these two hypotheses is calculated:

$$
\eta=\frac{\exp \left(-\frac{1}{2}\left(\hat{W}-b_{T} f_{j}\right)^{T} \hat{H}_{W}^{-1}\left(\hat{W}-b_{T} f_{j}\right)\right)}{\exp \left(-\frac{1}{2} \hat{W}^{T} \hat{H}_{W}^{-1} \hat{W}\right)}
$$

The calculation by choosing a test statistic [1] gives:

$$
P_{j}=b_{T}\left(2 f_{j}^{T} \hat{H}_{W}^{-1} \hat{W}-b_{T} f_{j}^{T} \hat{H}_{W}^{-1} f_{j}\right)
$$

The estimate $\hat{b}_{T}$ of $b_{T}$ is obtained by maximizing $P_{j}$ :

$$
\hat{b}_{T}=\left(f_{j}^{T} \hat{H}_{W}^{-1} f_{j}\right)^{-1}\left(f_{j}^{T} \hat{H}_{W}^{-1} \hat{W}\right)
$$

By replacing $\hat{b}_{T}$ in the expression (15), we obtain:

$$
P_{j}=\frac{\left(f_{j}^{T} \hat{H}_{W}^{-1} \hat{W}\right)^{p}}{f_{j}^{T} \hat{H}_{W}^{-1} f_{j}}
$$

The variable $P_{j}$, sum of squares of $n$ normally distributed variables follows a Chi 2 law with $n$ degrees of freedom. To detect and isolate the presence of a fault, we use the next statistic:

$$
P=\sup _{j} P_{j}>\chi_{n, 1-\alpha}^{2}
$$

The value of $P$ is compared to a threshold indexed by a confidence level $\alpha$. If $P$ is less than this threshold, measurement magnitudes are accepted and no temperature fault are detected. On the other hand, if $P$ is greater than this threshold, the variables belonging to an equation which normalized residual is greater than a threshold (fixed by the 
choice of the probability of error of type I) are declared faulty. These normalized residuals are computed as follows:

$$
\hat{W}_{N}=\left({D_{\hat{H}_{W}}}\right)^{2} \hat{W}
$$

where $D_{\hat{H}_{W}}$ represents the diagonal matrix containing diagonal terms of the matrix of variance-covariance $\hat{H}_{W}$.

The procedure of detection and isolation of flow and temperature measurement faults can be summarized by the following steps:

- step 1 - detection, isolation and estimation of flow measurement faults

Once we have isolated all flow measurement faults and estimated their magnitude by a well adapted technique for linear systems (method of parity space, method of measurement test, GLR method or others) which produce the same results, we proceed to the estimation of flow measurements. The estimation is given by the classical expression:

$$
\hat{Q}=\left(I_{v}-V_{Q} M_{Q}^{T}\left(M_{Q} V_{Q} M_{Q}^{T}\right)^{-1} M_{Q}\right)\left(Q-\hat{d}_{Q}\right)
$$

where $I_{v}$ is the identity matrix of dimension $v \times v$.

- $\quad$ step 2 - detection of suspicious temperature measurements

The normalized residual $W_{N}$ given by equation (19) is calculated. If no component of this vector exceeds the fixed threshold, the procedure is stopped. If the normalized residual of the $i$ th equation exceeds the threshold, the variables intervening in this equation are suspected to be faulty.

- step 3 - application of the GLR test to isolate suspicious temperature measurements

All the variables $P_{j}$ (equation (15)) associated to each suspected variables is therefore calculated. The variable $P$ given by the expression (18) is evaluated and compared to a threshold. If $P$ is greater than this value, the corresponding temperature measurement is declared faulty.

\section{- step 4 - estimation of the magnitude of temperature fault}

The magnitude of the temperature measurement fault is computed by using equation (16) ; the corresponding measurement is compensated and the residuals of bilinear constraint are evaluated again, this procedure is repeated from step 2 to detect eventual new temperature measurement faults.

- $\quad$ step 5 - data reconciliation of temperature measurements
After having determined temperatures that are faulty and estimated magnitude of their faults, we proceed to the estimation of temperature measurements.

The data reconciliation problem consists in determining the estimation $\hat{T}$ of the temperatures which minimizes the criterion:

$$
\Phi=\frac{1}{2}\left\|\hat{T}+\hat{d}_{T}-T\right\|_{V_{T}^{-1}}^{2}
$$

subject to: $\quad M_{T} D_{\hat{Q}} \hat{T}=0$

This problem can be solved by classical search of the stationary point of the associated Lagrangian. The estimation of temperature measurements is given by:

$$
\hat{T}=\left(I_{v}-V_{T} D_{\hat{Q}} M_{T}^{T} \hat{H}_{W}^{-1} M_{T} D_{\hat{Q}}\right)\left(T-\hat{d}_{T}\right)
$$

\section{Applications}

A cold flow $Q_{l}$ at a temperature $T_{l}$ is heated by a hot flow $Q_{8}$ at a temperature $T_{8}$ through heat exchangers.

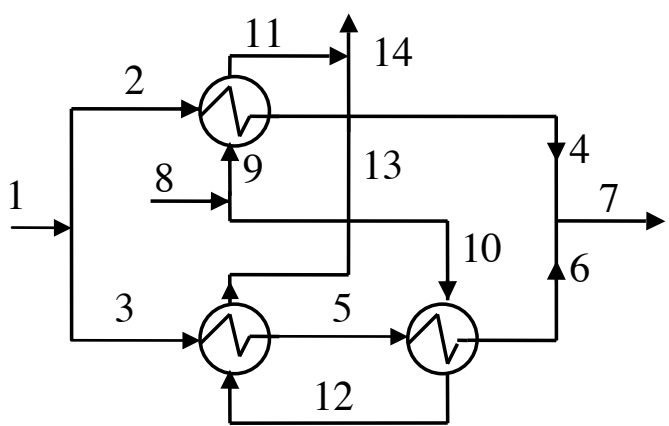

Figure 2: heat exchanger network

The incidence matrix of flow measurements is equal to:

$$
M=\left(\begin{array}{cccccccccccccc}
1 & -1 & -1 & 0 & 0 & 0 & 0 & 0 & 0 & 0 & 0 & 0 & 0 & 0 \\
0 & 1 & 0 & -1 & 0 & 0 & 0 & 0 & 0 & 0 & 0 & 0 & 0 & 0 \\
0 & 0 & 1 & 0 & -1 & 0 & 0 & 0 & 0 & 0 & 0 & 0 & 0 & 0 \\
0 & 0 & 0 & 0 & 1 & -1 & 0 & 0 & 0 & 0 & 0 & 0 & 0 & 0 \\
0 & 0 & 0 & 1 & 0 & 1 & -1 & 0 & 0 & 0 & 0 & 0 & 0 & 0 \\
0 & 0 & 0 & 0 & 0 & 0 & 0 & 1 & -1 & -1 & 0 & 0 & 0 & 0 \\
0 & 0 & 0 & 0 & 0 & 0 & 0 & 0 & 1 & 0 & -1 & 0 & 0 & 0 \\
0 & 0 & 0 & 0 & 0 & 0 & 0 & 0 & 0 & 1 & 0 & -1 & 0 & 0 \\
0 & 0 & 0 & 0 & 0 & 0 & 0 & 0 & 0 & 0 & 0 & 1 & -1 & 0 \\
0 & 0 & 0 & 0 & 0 & 0 & 0 & 0 & 0 & 0 & 1 & 0 & 1 & -1
\end{array}\right)
$$

and the incidence matrix $M_{T}$ is given by the following matrix: 


$$
M_{T}=\left(\begin{array}{cccccccccccccc}
0 & 1 & 0 & -1 & 0 & 0 & 0 & 0 & 1 & 0 & -1 & 0 & 0 & 0 \\
0 & 0 & 1 & 0 & -1 & 0 & 0 & 0 & 0 & 0 & 0 & 1 & -1 & 0 \\
0 & 0 & 0 & 0 & 1 & -1 & 0 & 0 & 0 & 1 & 0 & -1 & 0 & 0 \\
1 & -1 & -1 & 0 & 0 & 0 & 0 & 0 & 0 & 0 & 0 & 0 & 0 & 0 \\
0 & 0 & 0 & 1 & 0 & 1 & -1 & 0 & 0 & 0 & 0 & 0 & 0 & 0 \\
0 & 0 & 0 & 0 & 0 & 0 & 0 & 1 & -1 & -1 & 0 & 0 & 0 & 0 \\
0 & 0 & 0 & 0 & 0 & 0 & 0 & 0 & 0 & 0 & 1 & 0 & 1 & -1
\end{array}\right)
$$

One thousand observations whose measurement errors are normally distributed with zero mean and a known variance have been simulated. Afterwards, we have biased simultaneously the magnitude of the flow $Q_{1}$ of $+20 \%$, that of $Q_{11}$ of $+30 \%$ and that of the temperature $T_{11}$ of $+25 \%$ of their initial values.

The flow variables $Q_{1}, Q_{4}, Q_{6}, Q_{11}$ and $Q_{13}$ as well as the temperature variables $T_{1}, T_{4}, T_{5} T_{6}, T_{8}, T_{11}$, and $T_{13}$ are measured. Notice that all the unmeasured variables can be deduced as the system is observable. The table 1 shows simulated measurements of flow and temperature:

\begin{tabular}{|c|c|c|}
\hline $\begin{array}{c}\text { stream } \\
\text { number }\end{array}$ & $\begin{array}{c}\text { flow } \\
\text { measurement } \\
\text { t/day }\end{array}$ & $\begin{array}{c}\text { temperature } \\
\text { measurement } \\
{ }^{\circ} \mathrm{C}\end{array}$ \\
\hline 1 & $\underline{\mathbf{1 1 7 . 6}}$ & 81.8 \\
\hline 2 & 40.3 & 80.2 \\
\hline 3 & 61.8 & 81.1 \\
\hline 4 & 40.6 & 162.8 \\
\hline 5 & 59.4 & 131.1 \\
\hline 6 & 61.1 & 158.9 \\
\hline 7 & 99.5 & 153.4 \\
\hline 8 & 128.1 & 226.2 \\
\hline 9 & 79.3 & 217.8 \\
\hline 10 & 49.4 & 230.7 \\
\hline 11 & $\underline{\mathbf{1 0 3 . 4}}$ & $\underline{\mathbf{2 3 2 . 5}}$ \\
\hline 12 & 50.6 & 192.4 \\
\hline 13 & 48.9 & 137.2 \\
\hline 14 & 130.9 & 171.2 \\
\hline
\end{tabular}

Table 1: values of flow and temperature measurements

The different steps of detection, isolation and estimation are the following. In the first stage, the proposed strategy has been applied to detect and isolate the two faulty flow sensors. For a given threshold, the suspicious residuals due to the presence, in the flow data, of gross measurement errors can be detected. We can also estimate the magnitude of faults of these measurements and deduce the estimation of flow measurements 1 an 11 . The vectors of estimated magnitudes of faults and corrected flow measurements are:

$$
\begin{aligned}
& \hat{d}_{Q}=\left(\begin{array}{llllllllllllll}
24.1 & 0 & 0 & 0 & 0 & 0 & 0 & 0 & 0 & 0 & 16.7 & 0 & 0 & 0
\end{array}\right) \\
& \hat{Q}=\left(\begin{array}{lllllllll}
100.9 & 40.3 & 60.6 & 40.3 & 60.6 & 60.6 & 100.9 & 129.2 & 79.5
\end{array}\right. \\
& \left.\begin{array}{lllll}
49.6 & 79.5 & 49.6 & 49.6 & 129.2
\end{array}\right)^{\mathrm{T}} .
\end{aligned}
$$

The second stage consists in detecting and isolating the faulty temperature sensors. Applying the proposed strategy, we obtain:

$$
\begin{aligned}
\hat{d}_{T}= & \left(\begin{array}{llllllllllllll}
0 & 0 & 0 & 0 & 0 & 0 & 0 & 0 & 0 & 0 & 48.8 & 0 & 0 & 0
\end{array}\right) \\
\hat{T}= & \left.\begin{array}{llllllll}
81.4 & 80.8 & 81.8 & 159.2 & 129.3 & 156.9 & 157.8 & 225.6 \\
229.1 & 183.7 & 195.6 & 137.5 & 165.9
\end{array}\right)^{\mathrm{T}} .
\end{aligned}
$$

\section{Conclusions}

In this communication, we have extended the method of fault detection and localization based on the GLR test in the case of bilinear systems. The method has been applied to detect and isolate faults as well as to estimate flow and temperature measurements of heat exchanger systems.

In the first stage, the variables intervening in linear equations only are analyzed. The measurement faults of these variables are detected, isolated and their magnitudes are estimated. Thereafter, a classical method of estimation using projection matrix allows the corrected measurements to be obtained. After this step, a set of corrected flow measurements fitting the linear equations perfectly is obtained.

In the second stage, the GLR test is applied to bilinear constraint residuals computed using the previous corrected set of flow measurements. The test allows the gross measurement errors of temperature to be detected from the bilinear equations.

The results obtained from the heat exchanger example shows that the procedure simultaneously detects flow gross errors and temperature gross errors issued from the same stream. Analyzing the obtained results, we can say that this strategy is much more reliable than the pseudo node method [4] which was applied essentially in mineral processes. This method is based on the generation and analysis of all the residuals that can be obtained by elimination of common variables between two equations. Applied on the proposed example, the method of pseudo node was not able to detect and isolate correctly the measurement faults. In particular, the measurement faults on stream 11 (flow and temperature faults) were not correctly isolated. However, the pseudo node method is numerically more efficient because it needs less computation time.

\section{References}

[1] S. Narasimhan, R.S.H. Mah, "Generalized Likelihood Ratio Methods for Large Error Identification," AIChE Journal, vol. 33, $\mathrm{n}^{\circ}$, pp. 1514-1521, 1987. 
[2] R.S.H Mah., A.C. Tamhane, "Detection of Large errors in Process Data.," AIChE Journal, vol. 28,n5, pp. 828-831,1982.

[3] J. Ragot, A. Aitouche, F. Kratz, D. Maquin, " Detection and Localisation of Gross Errors in Instrument using Parity Space Technique, "International Journal of Mineral Processing, vol. 31,pp. 281-299, 1991.

[4] J. Ragot, D. Maquin, M. Darouach, "Analysis of Generalized Bilinear Systems. Application to Diagnosis.," Proceedings of IMACS-IFAC Symposium, Lille, France, 1991. 